\title{
SCHIZOPHRENIA AND OBSESSIVE-COMPULSIVE DISORDER: IS THERE EVIDENCE FOR A SCHIZO-OBSESSIVE DISORDER?
}

\author{
M. Araújo ${ }^{1}$, F. Caetano ${ }^{1}$, A. Samouco ${ }^{2}$, A. Norton ${ }^{1}$ \\ ${ }^{1}$ Hospital Magalhães Lemos ${ }^{2}$ Unidade Local de Saúde do Norte Alentejano \\ ULSRAF
}

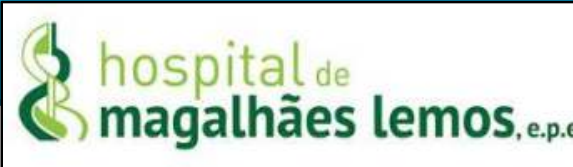

OBJECTIVES: We aim to review the epidemiology, clinical presentation and neurobiology of patients diagnosed with both obsessive-compulsive disorder (OCD) and schizophrenia (SZ), recently called schizo-obsessive disorder.

BACKGROUND AND AIMS: Co-occurrence of obsessive-compulsive symptoms (OCS) and OCD in schizophrenia has been described for years. Recent evidence suggests that this may represent more than mere comorbidity and the concept of a new clinical entity called "schizo-obsessive disorder" has been proposed. ${ }^{1}$

MATERIAL AND METHODS: Literature search through PubMed database applying the search terms: obsessive-compulsive disorder; psychosis; schizophrenia; schizo-obsessive disorder.

\section{RESULTS:}

\section{Epidemiology}

Instances of the co-occurrence of psychosis and OCS have been noted since the $19^{\text {th }}$ century with low prevalence rates $(1 \%-3.5 \%)$. However, recent studies revealed much higher comorbidity rates:

\begin{tabular}{|c|c|c|}
\hline Meta-analysis & $\begin{array}{c}\text { Prevalence rates for } \\
\text { OCD in SZ }\end{array}$ & $\begin{array}{c}\text { Prevalence rates for } \\
\text { OCS in SZ }\end{array}$ \\
\hline Buckley et al. $2019^{2}$ & $\mathbf{2 3 \%}$ & $25 \%$ \\
\hline Achim et al. $2011^{3}$ & $\mathbf{1 2 . 1 \%}$ & \\
\hline Swets et al. $2014^{4}$ & $\mathbf{1 2 . 3 \%}$ & $30.7 \%$ \\
\hline
\end{tabular}

- Among individuals considered at risk for developing psychosis, $11 \%$ exhibited OCS and 5\% OCD according to Zink et al. ${ }^{5}$

\section{IS THERE AN INCREASED RISK OF DEVELOPING SZ IN PEOLPLE DIAGNOSED WITH OCD?}

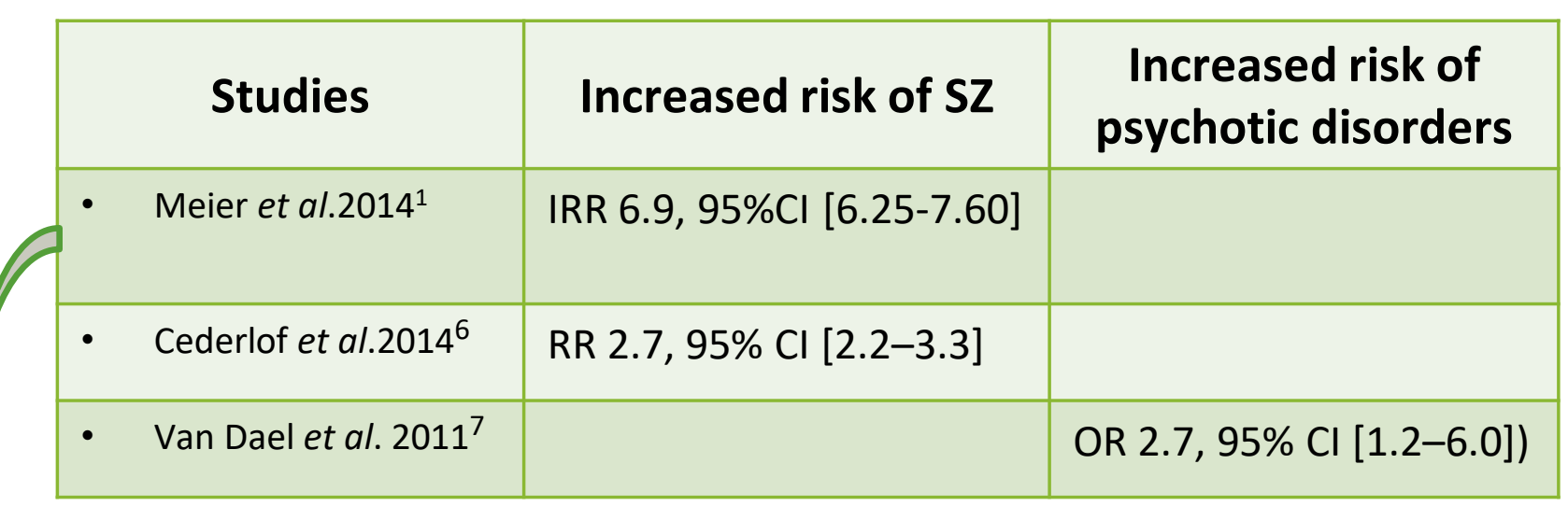

IRR - Incidence rate ratio; RR - Relative Risk; OR - Odds Ratio

This study also showed that offspring of parents diagnosed as having OCD had an increased risk of SZ and SZ spectrum disorders.

The remarkably high co-occurrence of OCD and OCS in SZ has resulted in a growing interest in the topic, and a new clinical entity called "schizo-obsessive disorder" was proposed.

\section{CLINICS}

OCS and OCD may appear at diferent stages of SZ and may manifest before and independently of psychosis, intermenttetly or latter in the ilness, prodromally and then consistently throughout the ilness.

\section{OCS may be:}

Strongly associated with the psychotic symptoms (SCHIZO-OBSESSIVE CONCEPT)
Proposed diagnostic criteria for schizo-obsessive disorder (Povurovsky et al) ${ }^{8}$

A. Symptoms are present that meet Criterion A for obsessive-compulsive disorder at some time point during the course of the schizophrenia.

B. If the content of the obsessions and/or compulsions is interrelated with the content of delusions and/or hallucinations, additional typical OCD obsessions and compulsions recognized by the person as unreasonable and excessive are required.

C. Symptoms of OCD are present for a substantial portion of the total duration of the prodromal, active and/or the residual period of schizophrenia.

D. The obsessions and compulsions are time-consuming, cause distress or significantly interfere with the person's normal routine, in addition to the functional impairment associated with schizophrenia.

E. The obsessions and compulsions are not due to the direct effect of antipsychotic agents, a substance of abuse or an organic factor.

\section{Compared to SZ, Schizo-obsessive patients show ${ }^{9,10:}$}

- Earlier onset of psychosis;

- More depressive symptoms and higher suicidality;

- Increased rates of hospitalization;

- Greater dysfunction and lower social functioning;

- Poor quality of life;

- Data regarding the positive and negative symptoms is conflicting, with some studies revealing no significant differences between groups.

Obsessive-compulsive symptoms are comonly severe and tend to appear during the prodromal stages of psychosis.

\section{Neurobiology}

Although very few neuroimaging studies on schizo-obsessive disorder are available, some studies found: ${ }^{11}$

- Reduced volumes of the left hippocampus and frontal lobes;

- Alterations of the anterior horn of the lateral and third ventricles;

- Abnormalities in the frontal areas and caudate nucleus (functional imaging studies).

Whether these findings reflect a specific pattern of dysfunction unique to this comorbid group or a severer form of $\mathrm{SZ}$ is unclear. ${ }^{11}$

In relation to neuropsychological performance, contradictory findings have been reported, with some studies showing poorer results in the schizoobsessive group. ${ }^{9}$

CONCLUSIONS: Evidence suggests that patients with comorbid OCD and SZ may represent a special category among patients with schizophrenia. Although clinical aspects are more consensual, data regarding neuroimaging and neuropsychological features are scarce and further neurobiological research is needed to elucidate if this subtype of the SZ population represents a specific diagnostic entity. 